\title{
KSHV Down-regulates Tropoelastin in Both an in-vitro and in-vivo Kaposi's Sarcoma Model
}

\begin{abstract}
Keywords: Kaposi's sarcoma; Extracellular matrix; Fibronectin; Tumor model; Biomarkers

Abstract

Kaposi's sarcoma (KS), a common cancer in individuals with HIV/ AIDS, lacks a curative therapy. Few studies have examined changes in extracellular matrix (ECM) protein profiles in the development of KS. Here we used an in vitro (human demal microvascular endothelial cells, DMVEC) and an in vivo mECK mouse model of Kaposi's to study the impact of infection on tropoelastin. Using DMVEC, Kaposi's sarcoma-associated herpesvinus (KSHV) reduced tropoelastin transcription when examined at 2, 5, 7, and 10 days post addition, a finding that was inversely correlated with a rise in viral latency associated nuclear antigen (LANA) transcription. Immunohistochemical/immunofluorescence data confirmed that DMVEC cells were KSHV-infected (evidenced by LANA production) and that there was a loss of tropoelastin protein compared to controls. Using the mECK36 mouse model of KS we observed a reduced expression of tropoelastin mRNA in 3 of 3 tumor biopsies compared to controls. Immunofluorescence staining showed high levels of viral LANA expression in the tumor core, while immunohistochemical staining showed high levels of LANA expression and spindle cells in tumors. Dual label immunohistochemistry on formalin-fixed paraffinembedded tumor tissue revealed reduced expression of tropoelastin in LANA positive spindle cell regions quantified by Ariol SL-50 scanning analysis. Together, this suggests that alterations in tropoelastin may play an important role in the development of Kaposi's and could serve as an early marker of this disease. This information will also allow us to explore the potential role of tropoelastin anti angiogenic properties in an in vivo model for $\mathrm{KS}$ disease.
\end{abstract}

\section{Introduction}

Kaposi's sarcoma-associated herpes virus (KSHV) or Human Herpes virus type 8 (HHV8) is the causal agent of Kaposi's sarcoma (KS) $[1,2] . \mathrm{KS}$ is an angioproliferative malignancy of vascular endothelial cells and produces B cell lymphoproliferative diseases in the form of pleural effusion lymphomas (PEL) and some forms of Multicentric Castleman's Disease [3,4]. Kaposi's sarcoma is now the predominant HIV/AIDS related malignancy in Southern Africa and hence the world [5]. Antiretroviral therapy has significantly reduced the incidence of KS in Western countries however $95 \%$ of the estimated 33-40 million people infected with HIV have no access to these therapeutic drugs. Organ transplant patients are also at risk for developing KS [6,7], while classical KS seen in elderly males of Mediterranean or eastern European descent remains to be a problem [8]. Currently there are no curative therapies for KS. In addition, although antiretroviral drugs have been shown to induce regression of KS, only $50 \%$ of treated patients have complete remission of the disease $[9,10]$. Radiotherapy has been used for isolated lesions and systemic chemotherapy for disseminated disease with limited success. Also there is poor access to these therapies globally.

\section{Journal of $\varnothing$ Oncobiomarkers}

\section{Donald J. Alcendor*}

Center for AIDS Health Disparities Research and the Department of Microbiology and Immunology, School of Medicine, Meharry Medical College, Nashville, Tennessee, USA

\section{Address for Correspondence}

Donald J. Alcendor, Center for AIDS Health Disparities Research and the Department of Microbiology and Immunology, School of Medicine, Meharry Medical College, Nashville, Tennessee, USA, Tel: (+1) 615327-6449; Fax:(+1) 615-327-6929; E-mail: dalcendor@mmc.edu

Copyright: ( $) 2015$ Alcendor DJ. This is an open access article distributed under the Creative Commons Attribution License, which permits unrestricted use, distribution, and reproduction in any medium, provided the original work is properly cited.

Submission: 02 June 2015

Accepted: 19 June 2015

Published: 23 June 2015

Reviewed \& Approved by: Dr. Youming Xie, Associate Professor Department of Oncology \& Karmanos Cancer Institute, Wayne State University School of Medicine, Detroit, USA

While we know very little about the pathogenic progression of Kaposi's sarcoma, the extracellular matrix (ECM) is known to play a critical role in regulating cellular functions such as cell adhesion, proliferation and cell migration in other malignancies [11]. There have been only a few studies that have examined changes in ECM protein profiles in KS lesions. These primarily have focused on matrix metalloproteinase (MMPs) [12-16]. We also previously reported that fibulins $-2,-3$, and -5 were significantly decreased in DMVEC cells exposted to KSHV, fibulins 1C/1D were increased and fibulins $-4,-6$, and -7 were unchanged [17]. As part of that study, we looked briefly at fibulin-2 binding partners using DMVEC cells, and found that both tropoelastin (25-fold) and fibronectin (5-fold) were decreased 10 days following addition of KSHV, relative to untreated controls. As tropoelastin is an important ECM protein that provides strength and elasticity to the skin and other organs and is critical for wound healing, we decided to study this ECM protein in more detail. Soluble forms of elastin (tropoelastin) as well as elastin peptides have been shown to be potent inhibitors of metastasis in experimental tumor models suggesting an important role for tumor-cell elastin interactions in tumor progression [18-20].

This is the first study to examine the role of tropoelastin expression in KSHV infected DMVEC cells and in an in vivo animal model of Kaposi's. This study could provide the basis for the identification of an ECM biomarker useful for early diagnosis and/or disease progression as well as a novel target for treatment of KS.

\section{Materials and Methods}

All human and animal studies were approved by Meharry Medical College's Institutional Review Board and IACUC.

\section{mECK36 mice and tumors biopsies}

Mouse tumor biopsies from mECK animals and corresponding fresh frozen OTC preserved tissue on slides from the KSHV induced KS mouse model was kindly provided Enrique Mesri from Sylvester Comprehensive Cancer Center, Miami Florida. The development of this model has been previously described by Mutlu et al. [21].

\section{Cells and viruses}

The BCBL-1 cell line, originally isolated from a body cavity-based 
human lymphoma, was cultured in RPMI 1640 media (Gibco, Grand Island, NY) until the cell density reach $3 \times 10^{6}$ cells/flask. Then lytic cycle virus replication was induced with TPA (12-O-Tetradecanoylphorbol-13-acetate) at $20 \mathrm{ng} / \mathrm{ml}$ and sodium butyrate at $0.3 \mathrm{ng} / \mathrm{ml}$. Twenty-four hours, post induction cells were washed twice in PBS to remove butyrate, and induction was continued with TPA for 5 days. Cell free virus was isolated and concentrated by differential centrifugation. Dermal microvascular endothelial cells (DMVEC) were maintained in complete EMB-2 media Lonza, Basel, Switzerland) and at passage level 4 they were infected at a MOI of 0.01 . Mock infected cells were used as controls. Ten days post infection cells were prepared for immunofluorescent staining.

\section{Giemsa staining}

Normal and KSHV infected DMVEC cells were cultivated in chamber slides at $80 \%$ confluence. Media was removed and cells were washed three times with phosphate buffer saline (PBS) pH 7.4, then fixed for $15 \mathrm{~min}$ in absolute methanol at $-20^{\circ} \mathrm{C}$. Giemsa stock stain was diluted in Giemsa buffer and cells were stained according to the manufacturer's recommendations (Invitrogen, Carlsbad, CA). Stained cells were dried and sealed with a glass cover slip using permanent mounting medium. Slides were viewed on a Nikon TE2000S microscope mounted with a charge-coupled device camera under bright-field illumination at a total magnification of 200x.

\section{RNA and cDNA ampification}

Total RNA was extracted from KSHV infected DMVEC cells and mock infected cells using a Qiagen mini RNA isolation kit (Qiagen, Valencia, CA). The RNA was DNAase treated prior to elution on the column according to the manufacturer's recommendations. Messenger RNA in one microgram of each sample was primed using oligo-dT and reverse transcribed with a High Capacity cDNA reverse transcription kit (Applied Biosystems, Foster City, CA.).

\section{Real-Time qPCR}

Real Time PCR was performed in 96 well optical plates (Sorenson Bioscience, Inc.) with cDNA using the MyiQ Single Color Real Time PCR Detection System (Bio-Rad Laboratories, Hercules, CA) in $25 \mu \mathrm{l}$ reaction volumes. A master mix was made according to manufacturer's instructions using SYBR Green Supermix (Bio-Rad Laboratories, Hercules, CA) or Veriquest master mix (Affymetrix, Santa Clara, CA) for amplification of high GC content cDNAs. Forward and reverse primers were used at a concentration of 250 $n M$ per well in RNAase, DNAase free $\mathrm{H}_{2} \mathrm{O}$. Primer sequences for qPCR were as follows: tropoelastin forward 5'-GAGTGAAGCCTG GGAAAGTG-3', reverse 5'-CCAGCAAAAGCTCCACCT AC-3'; KSHV LANA forward 5'-CCTCCATCCCATCCTGTGTC -3', reverse 5'- GGACGCATAGGTGTTGAAGAG-3'); and GAPDH forward 5'-GAAGGTGAAGGTCGGAGT-3', reverse 5'-GAAGATGGTGAT GGGATTTC. The cDNAs from mock infected and KSHV infected DMVEC cells were diluted 1:3 using RNAase DNAase free $\mathrm{H}_{2} \mathrm{O} ; 3$ $\mu \mathrm{l}$ of this dilution was added to each well. Control wells substituted water for cDNA. The cycling sequence included $95^{\circ} \mathrm{C}$ for $3 \mathrm{~min}, 95$ ${ }^{\circ} \mathrm{C}$ for $15 \mathrm{~s}, 60^{\circ} \mathrm{C}$ for $1 \mathrm{~min}, 95^{\circ} \mathrm{C}$ for $1 \mathrm{~min}, 55^{\circ} \mathrm{C}$ for $1 \mathrm{~min}$, and 55 ${ }^{\circ} \mathrm{C}$ for $30 \mathrm{~s}$ for 81 total cycles. A GAPDH primer set was included for normalization. Data analysis was performed using Bio-Rad iQ5 Optical System Software Version 2.

\section{Immunohistochemistry and immunofluorescence}

Immunohistochemistry for KSHV LANA and PECAM-1/CD34 was performed on nodular archival human AIDS-KS tissue fixed in formalin, embedded in paraffin, sectioned and placed on chemate slides prior to immunohistochemistry. Immunohistochemistry for tropoelastin and KSHV LANA were also performed on formalin fixed paraffin embedded mouse tumor tissue. For staining, antigen retrieval was performed with a microwave oven pretreatment (500 watts) in citrate buffer at $95^{\circ} \mathrm{C}$ for 15 minutes. Cells were then incubated $30 \mathrm{~min}$ in PBS pH 7.2, containing $10 \%$ normal goat serum with $1 \%$ Tween- 20 for blocking of nonspecific binding and permeabilization. Cells were then incubated with a rabbit polyclonal antibody to PECAM-1/ CD31 (Novus Biologicals, Littleton, CO) diluted 1:100 in blocking buffer. After 90 min of incubation, cells were subsequently washed, incubated with a biotinylated donkey anti-rabbit IgG antibody (DAKO) at a 1:100 dilution and then with a horseradish peroxidase-avidin conjugate (DAKO) at 1:500 dilution. Color development was achieved by incubating cells with the substrate 3,3-diamino-benzidine (DAB, SIGMA). The second labeling was achieved by an additional antigen retrieval step in the microwave as described above and a second round of staining with a monoclonal antibody to the KSHV LANA (Vector Laboratories, Burlingame, CA) at a 1:100 dilution. Cells were subsequently washed, incubated with a biotinylated goat anti-mouse/ rabbit IgG antibody (DAKO) at a 1:100 dilution and then an alkaline phosphatase-strepavidin conjugate (Vector Labs, Burlingame, CA) at a 1:500 dilution. Color development was achieved by incubating cells with the substrate vector red using a vector red alkaline phosphate substrate kit (Vector Laboratories, Burlingame, CA) according to the manufacturer's instructions. Images were taken with a Nikon TE2000 microscope mounted with a charge-coupled device camera.

For immunofluorescence analyses, OTC embedded tissue sections obtained from mECK tumor biopsies on slides were air dried and placed in acetone for $1 \mathrm{~min}$ to remove fixative. Cells were air dried for $15 \mathrm{~min}$, hydrated in Tris saline buffer ( $\mathrm{pH}$ 7.4) for $5 \mathrm{~min}$, and then incubated for $1 \mathrm{~h}$ at $37^{\circ} \mathrm{C}$ with monoclonal antibodies to KSHV LANA at a 1:50 dilution in PBS pH 7.4 (Vector Laboratories, Burlingame, CA). Cells were washed 3 times with Tris saline and then incubated at $37^{\circ} \mathrm{C}$ for 30 min with secondary donkey anti-mouse IgG antibodies conjugated with fluorescein isothiocyanate [(FITC), Jackson ImmunoResearch, West Grove, PA] at a 1:100 dilution in PBS. Cells were washed another 3 times in Tris saline and mounted with Vectashield mounting media (Burlingame, CA) containing $1.5 \mu \mathrm{g} / \mathrm{ml}$ of 4',6-diamidino-2-phenylindole (DAPI). Fluorescence was photographed with a Nikon TE 2000 S fluorescent microscope mounted with a charged coupled-device camera.

\section{Ariol SL-50 analyses}

Ariol SL-50 IHC scanning analysis platform from Genetix was employed to analyze dual labeled IHC stained mECK36 tumors $[22,23]$. The unit offers high resolution even multicolored imaging that can be exported in different file formats. The imaging software detects tissue directly on the slide and images can be rescanned automatically at different magnifications after IHC staining. Results are presented in a color/field area of red and green to indicate staining intensity for KSHV LANA and tropoelastin. 


\section{Statistical analyses}

Experiments shown in this study were performed in triplicate and quantitative real-time PCR (qRT-PCR) experiments were replicated three times and normalized to GAPDH [24].

\section{Results}

\section{KSHV infection of DMVEC}

Uninfected DMVEC cells displayed a cobblestone appearance as seen by phase microscopy and Giemsa staining which is consistent with the morphology of normal early passaged DMVEC cells (Figures 1a and b). These normal DMVEC were infected in vitro with BCBL1 virus and after 10 days post infection showed distinct morphological changes consistent with the spindle cell phenotype for KSHV infected DMVEC (Figure 1c) and KS tumors in vivo [17]. This swirling cell pattern was more pronounced when infected cells were stained with Giemsa stain (Figure 1d). We observed a similar spindle cell morphology in a human AIDS-KS tumor specimen with nuclei (brown) positively stained for KSHV LANA (Figure 1e). Dual labeled IHC staining for the endothelial cell marker PECAM-1/CD31 (brown) and KSHV LANA (red) showed a reduced staining of the CD31 in regions positive for KSHV LANA (Figure 1f).

\section{KSHV downregulates tropoelastin in infected DMVEC, a finding which correlates with an increase in KSHV LANA transcription}

We examined the temporal expression of tropoelastin in KSHV infected DMVEC by a time course transcriptional analysis using qRT-PCR. At 2 days post infection we observed a marginal but insignificant up regulation of mRNA expression in KSHV infected cells (Figure 2a).

However at 5, 7 and 10 days post infection, there was a consistent decrease in tropoelastin mRNA expression in KSHV infected cells compared mock infected controls. Over the same time frame, we observed a consistent increase in LANA transcription in KSHV infected cells compared to mock infected control cells (Figure 2b). This suggests that virus-induced changes in tropoelastin were inversely correlated with the time course of latent viral gene expression.

\section{Tropoelastin transcription and expression is suppressed in tumor tissue from a murine model for KSV-induced KS}

We examined transcriptional expression of KSHV LANA and tropoelastin by qRT-PCR in the mECK36 murine model for KSHVinduced KS [21]. Using RNA extracted from mouse tumor biopsies, we observed high levels of KSHV LANA transcription in 3 of 3 biopsies from different experimental animals when compared to RNA extracted from skin biopsies of 3 normal untreated animals (Figure 3a). Transcriptional analysis of tropoelastin levels was performed using RNA from mECK36 and control mouse tissues. We found increased levels of tropoelastin in normal dermal tissue from 3 of 3 animals when compared to tumor tissue in 3 of 3 animals (Figure $3 \mathrm{~b}$ ). We also examined fresh frozen OTC embedded tumor tissue by IFA from a mECK36 tumor- bearing mouse. Results showed low levels of LANA expression (green punctuate staining pattern, Figure 4a) at the periphery of the tumor core and very high levels of LANA staining in the tumor core (Figure 4b). By IHC staining of FFPE (formalinfixed, parafin-embedded) mouse tumor tissue we show characteristic
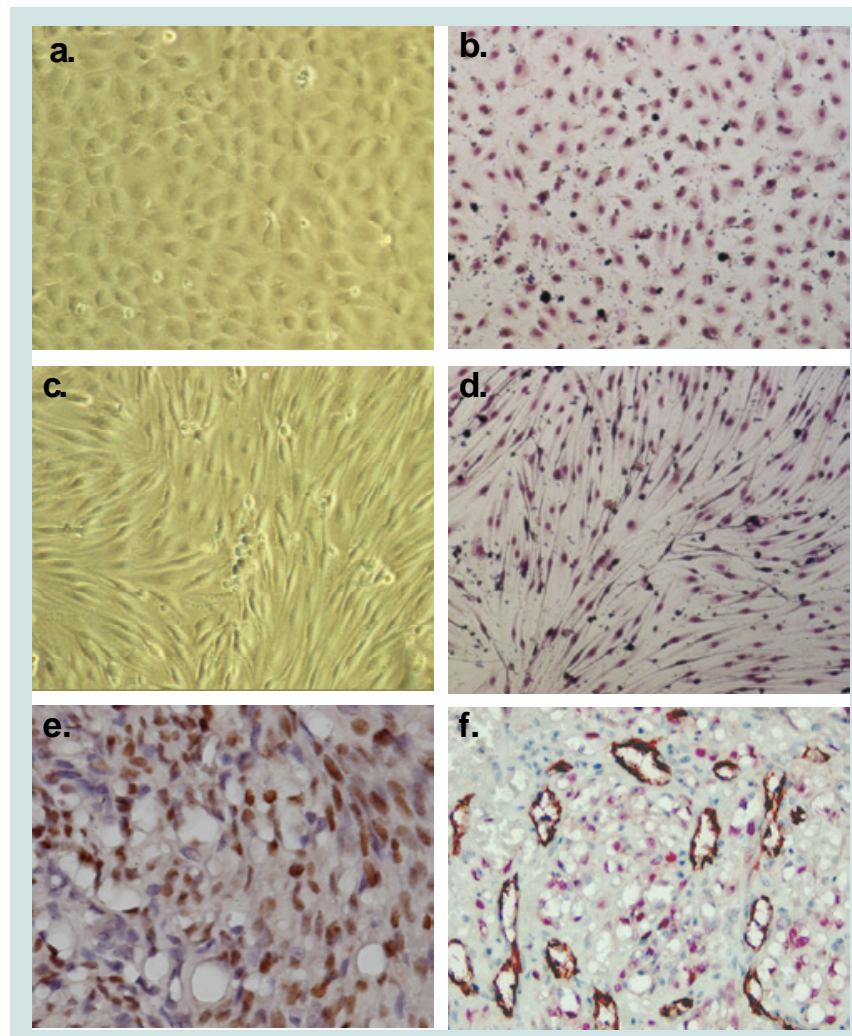

Figure 1: KSHV Infection of DMVEC Cells and KS Tissue.

LANA positive spindle cells staining brown (Figure 4c). LANA expression was observed as a nuclear punctuate staining pattern (Figure 4d). Dual labeled IHC staining of tumor sections revealed reduced tropoelastin staining (brown) in LANA positive (red) spindle cell regions (Figure 4e). We also showed a normal staining pattern and intensity for tropoelastin in tumor tissue in the absence of LANA positive cells (Figure $4 \mathrm{f}$ ). In addition, we showed a significant reduction of tropoelastin staining (brown) in LANA positive (red) spindle cell regions (Figures $4 \mathrm{~g}$ and $4 \mathrm{~h}$ ). Quantitative analysis of tropoeleastin (green) and LANA (red) was performed using an Ariol SL-50 slide scanner dual stained by immunohistochemistry (Figure $5 a)$. Therefore in a characteristic LANA positive spindle region (red) we observed a reduced expression of tropoelastin as well as higher levels of LANA staining in the tumor (indicated by the dotted circle in the upper and lower panels; Figure 5b).

\section{Discussion}

\section{DMVEC in-vitro model}

The ECM plays a key role in establishment, maintenance and progression of tumors. However its role in KS pathogenesis is poorly understood. This study is a continuation of our previous studies that link dysregulation of yet another important ECM protein, namely tropoelastin, to viral replication and tumorigenesis. We previously reported that ECM proteins fibulin-2, fibulin-3, and fibulin-5 as being transcriptionally downregulated in KSHV infected DMVEC cells [17]. We then examined several fibulin ECM binding partners and observed that tropoelastin was downregulated 25-fold in KSHV infected DMVEC cells [17]. 
a.

Temporal Expression of Tropoelastin by qRT-PCR in KSHV Infected DMVEC Cells

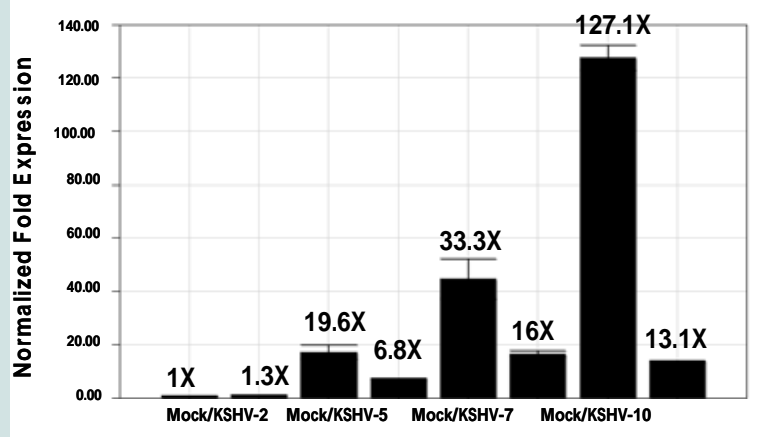

b.

Temporal LANA Expression by qRT-PCR in 10 Day KSHV Infected DMVEC Cells

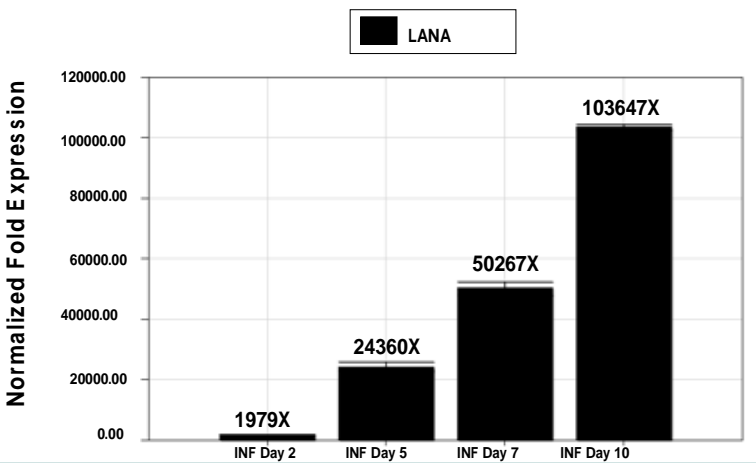

Figure 2: A) Temporal Expression of Tropoelastin by qRT-PCR in KSHV Infected DMVEC Cells. B) Temporal LANA Expression by qRT-PCR in 10 Day KSHV Infected DMVEC Cells.

a. Expression of KSHV LANA by qRT-PCR in Biopsies from Nomal Tissue and mECK36 Mouse Tumor Tissue

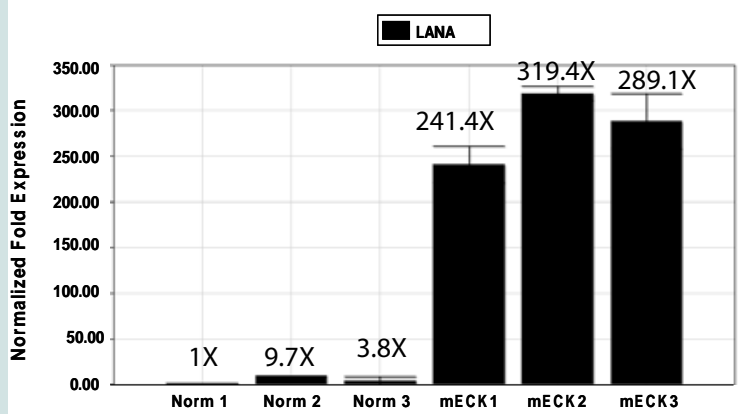

b. Expression of Tropoelastin by qRT-PCR in Biopsies from Normal Tissue and mECK36 Mouse Tumor Tissue Tropoelastin

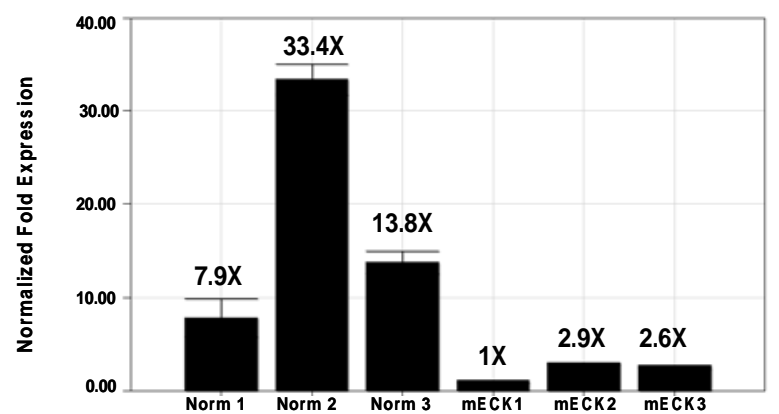

Figure 3: A) Expression of KSHV LANA by qRT-PCR in Biopsies from Normal Tissue and mECK36 Mouse Tumor Tissue. B) Expression of Tropoelastin by qRTPCR in Biopsies from Normal Tissue and mECK36 Mouse Tumor Tissue.

A time course analysis in this study revealed there was a sustained tropoelastin suppression over time of KSHV infection in DMVEC cells that correlated with an increase in viral replication (LANA) which suggests that tropoelastin could have an important role in progression of the pathogenesis of KSHV [17].

\section{The mECK36 KSHV dependent mouse model}

The bacterial artificial chromosome encoding the KSHV genome (KSHV BAC36) was used by Dr. Mesri, University of Florida, Miami, to transfect mouse bone marrow endothelial cells to generate the mECK36 cell line that forms KS-like tumors in mice [21]. The mECK36 cell line was found to express most known KSHV genes in vitro and when injected into nude mice produced vascularized spindle-cell sarcomas that were LANA+/podoplanin+, overexpressed VEGF, expressed angiopoietin ligands and receptors, and displayed KSHV and host transcriptomes reminiscent of KS [21]. The viral and host cell genes expression profiles (comparing orthologs in the mouse to humans) are similar in mECK tumors and KS. The KSHV transcriptome in mECK36 tumors and the mECK36 endothelial cells includes expression of KSHV latency genes [21]. mECK36 mouse tumors have phenotypic and molecular characteristics that make it suitable for studies analyzing the role of viral and host genes in KS and for preclinical testing of anti-KS drugs and routes of exposure [19]. Here we utilized tumor tissue from the mECK-36 KSHV dependent mouse model for KS to determine if KS-like lesions in whole tumor biopsies would reveal suppression of tropoelastin mRNA expression. We observed consistent transcriptional downregulation of tropoelastin mRNA in 3 of 3 whole tumor biopsies all shown to express high levels of KSHV LANA compared biopsies of normal skin from uninfected animals (Figure 3). Tropoelastin protein expression was also found to be downregulated by dual labeled immunostaining at sites in the tissue positive for LANA expression by quantitative analysis using the Ariol SL-50 scanning platform. This information provides an opportunity to determine the in vivo significance of tropoelastin suppression in an important model system for KS disease. Downregulation of fibulins 2, fibulin-5, fibulin-3, tropoelastin and fibronectin in KSHV infected DMVEC cells could translate in vivo to defects in elastic fiber formation in the endothelium, leading to an increase in vascular injury and increased angiogenesis that would in turn promote tumor progression. Changes in these proteins may also reflect the extent of tumor progression. These studies may lead to the identification of an ECM biomarker useful for early diagnosis and/ 


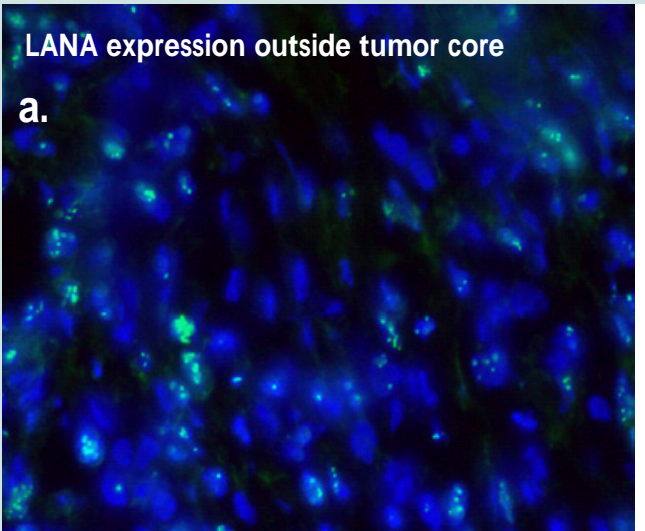

LANA+ spindle cells $400 \mathrm{X}$

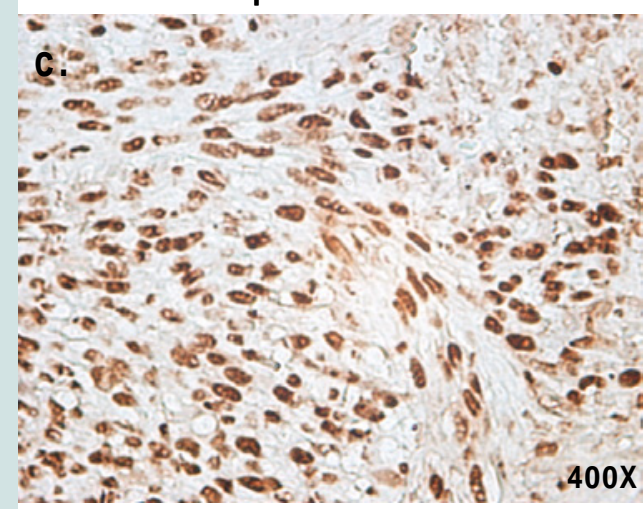

mECK36 tumor with LANA+ Regions 40X

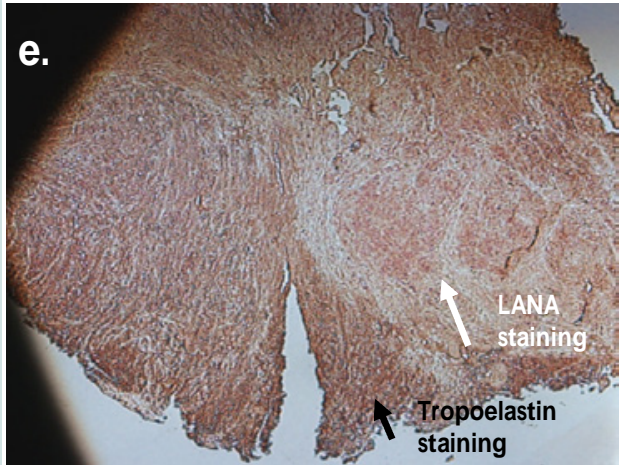

mECK36 LANA+ spindle cells (red) 200X

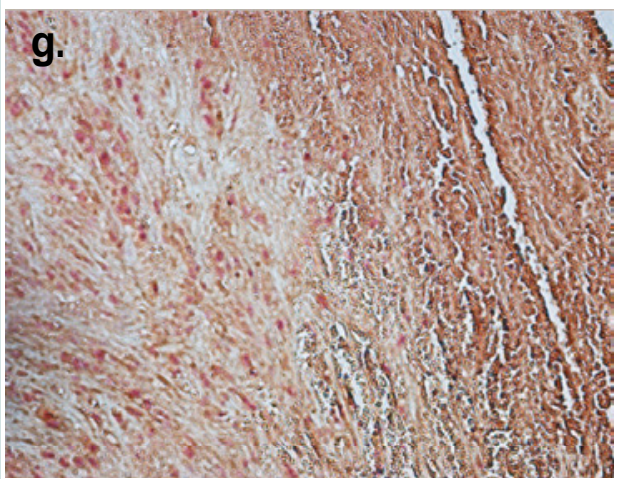

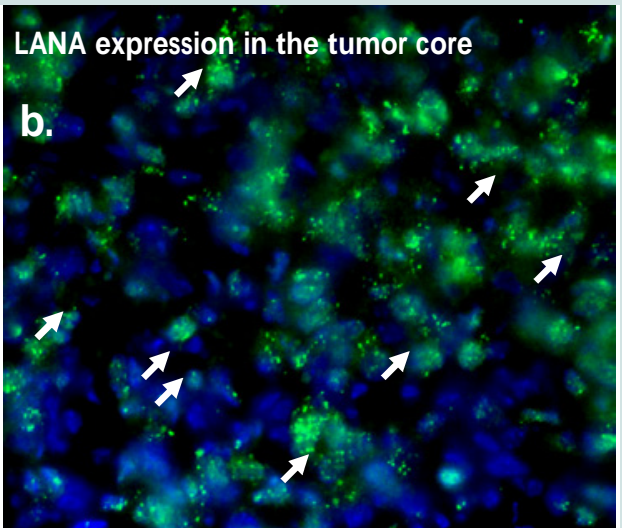

\section{LANA punctate nuclear staining pattem $600 \mathrm{X}$}

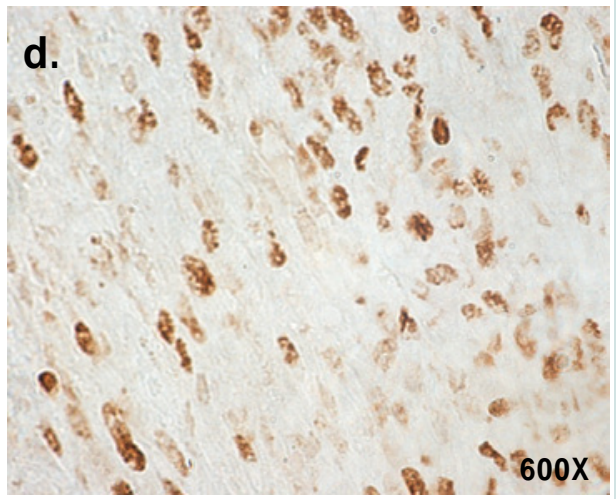

mECK36 for tropoelastin (brown) 200X

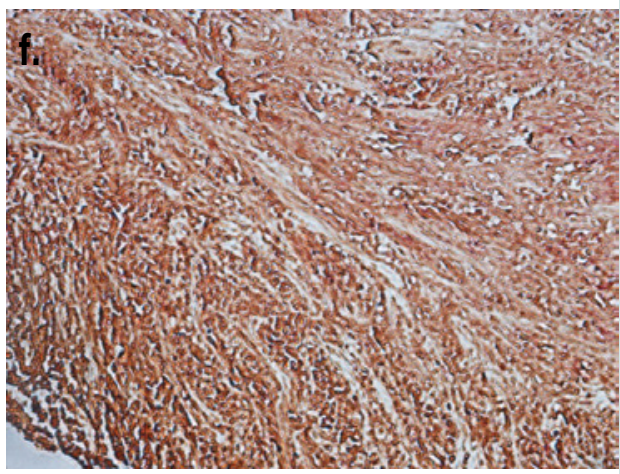

mECK36 LANA+ spindle cells (red) 400X

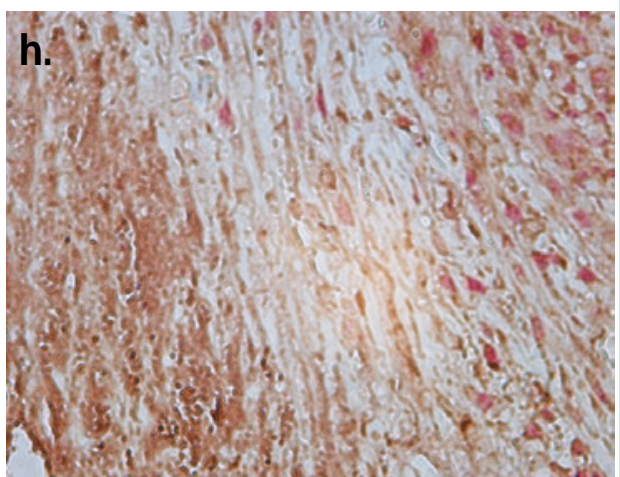

Figure 4: IFA and IHC Staining for KSHV LANA and Tropoelastin in Sarcomas of the mECK model for KSHV Induced KS. 

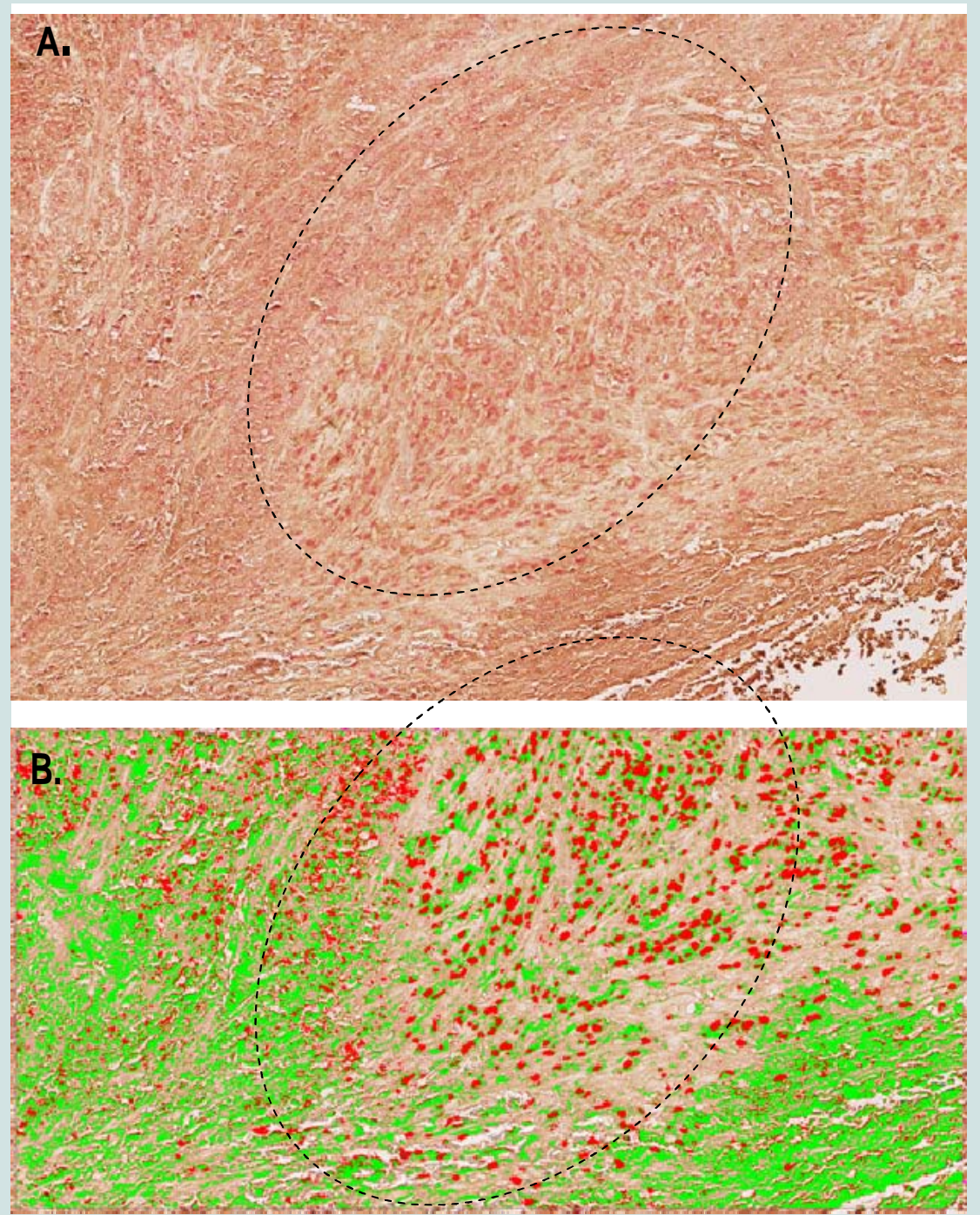

Figure 5: Dual Labeled IHC Staining for KSHV LANA and Human Tropoelastin using Ariol SL-50 Scanning of Mouse Tumor Tissue.

or disease progression. This information will also allow us to explore the potential role of tropoelastin in an in vivo model for KS disease.

\section{References}

1. Gramolelli S, Schulz TF (2015) The role of kaposi sarcoma-associated herpesvirus in the pathogenesis of kaposi sarcoma. J Pathol 235: 368-380.

2. Cavallin LE, Goldschmidt-Clermont P, Mesri EA (2014) Molecular and cellular mechanisms of KSHV oncogenesis of Kaposi's sarcoma associated with HIV/ AIDS. PLoS Pathog 10: e1004154.

3. Kedes DH, Operskalski E, Busch M, Kohn R, Flood J, et al. (1996) The seroepidemiology of human herpesvirus 8 (Kaposi's sarcoma-associated herpesvirus): distribution of infection in KS risk groups and evidence for sexual transmission. Nat Med 2: 918-924.

4. Schultz TF (2000) Kaposi's sarcoma-associated herpesvirus (human herpesvirus 8): epidemiology and pathogenesis. J Antimicrob Chemother 45 Suppl T3: 15-27.

5. Sitas F, Newton R (2001) Kaposi's sarcoma in South Africa. J Natl Cancer Inst Monogr 28: 1-4.

6. Alkharsah KR, Alzahrani AJ, Obeid OE, El-Harith EH, Guella A, et al. (2014) Vascular endothelial growth factor A polymorphism and risk of Kaposi's sarcoma herpesvirus viremia in kidney allograft recipients. Transpl Infect Dis 16: 783-789

7. Zafar SY, Howell N, Gockerman JP (2008) Malignancy after solid organ transplantation: an overview. Oncologist 13: 769-778.

8. Goedert JJ, Vitale F, Lauria C, Serraino D, Tamburini M, et al. (2002) Risk factors for classical Kaposi's sarcoma. J Nat Cancer Inst 94: 1712-1718.

9. Bower M, Palmieri C, Dhillon T (2006) AIDS-related malignancies: changing epidemiology and the impact of highly active antiretroviral therapy. Curr Opin Infect Dis 19: 14-19.

10. Monini P, Sgadari C, Toschi E, Barillari G, Ensoli B (2004) Antitumor effects of antiretroviral therapy. Nat Rev Cancer 4: 861-875.

11. Campbell NE, Kellenberger L, Greenaway J, Moorehead RA, Linnerth-Petrik NM, et al. (2010) Extracellular matrix proteins and tumor angiogenesis. J Oncol 2010: 1-13.

12. Bongiorno MR, Doukaki S, Ferro G, Arico M (2010) Matrix metalloproteinases 2 and 9, and extracellular matrix in Kaposi's sarcoma. Dermatol Ther 23 Suppl 2: S33-S36.

13. Pantanowitz L, Dezube BJ, Hernandez-Barrantes S, Tahan SR, Dabbous MK (2006) Matrix metalloproteinases in progression and regression of Kaposi's sarcoma. J Cutan Pathol 33: 793-798. 
Citation: Alcendor DJ. KSHV Down-regulates Tropoelastin in Both an in-vitro and in-vivo Kaposi's Sarcoma Model. J Oncobiomarkers. 2015;2(1): 7.

14. Meade-Tollin LC, Way D, Witte MH (1999) Expression of multiple matrix metalloproteinases and urokinase type plasminogen activator in cultured Kaposi's sarcoma cells. Acta Histochem 101: 305-316.

15. Kaaya EE, Castanos-Velez E, Amir H, Lema L, Luande J, et al. (1996) Expression of adhesion molecules in endemic and epidemic Kaposi's sarcoma. Histopathology 29: 337-346.

16. Becker J, Schuppan D, Reichart P (1987) The extracellular matrix in ora Kaposi's sarcoma (AIDS): the immunohistochemical distribution of collagens type IV, V, VI, of procollagens type I and III, of laminin and of undulin Virchows Arch A Pathol Anat Histopathol 412: 161-168.

17. Alcendor DJ, Knobel S, Desai P, Zhu W, Hayward GS (2011) KSHV Regulation of fibulin-2 in Kaposi's sarcoma: Implications for tumorigenesis. Am J Pathol 179: 1443-1454.

18. Tsuruga E, Yajima T, Irie K (2004) Induction of fibulin-5 gene is regulated by tropoelastin gene, and correlated with tropoelastin accumulation in vitro. Int $\mathrm{J}$ Biochem Cell Biol 36: 395-400.

19. Vrhovski B, Weiss AS (1998) Biochemistry of tropoelastin. Eur J Biochem 258: $1-18$
20. Schwartz E, Feinberg E, Lebwohl M, Mariani TJ, Boyd CD (1995) Ultraviolet radiation increases tropoelastin accumulation by a post-transcriptional mechanism in dermal fibroblasts. J Invest Dermatol 105: 65-69.

21. Mutlu AD, Cavalin LE, Vincent L, Chiozzini C, Eroles $P$, et al. (2007) In vivorestricted and reversible malignancy induced by human herpesvirus-8 KSHV: a cell and animal model of virally induced Kaposi's sarcoma. Cancer Cell 11: 245-258.

22. Alcendor D, Knobel S (2010) Identifying dysregulated genes induced by Kaposi's sarcoma-associated herpesvirus (KSHV). J Vis Exp.

23. Fiore C, Bailey D, Conlon N, Wu X, Martin N, et al. (2012) Utility of multispectral imaging in automated quantitative scoring of immunohistochemistry. J Clin Pathol 65: 496-502.

24. Alcendor DJ, Charest AM, Zhu WQ, Vigil HE, Knobel SM (2012) Infection and upregulation of proinflammatory cytokines in human brain vascular pericytes by human cytomegalovirus. Neuroinflammation 9: 95.

\section{Acknowledgements}

We thank Dr. Enrique Mesri for providing specimens from the $\mathrm{mECK} / \mathrm{KSHV}$ mouse model. We acknowledge the advice of James EK Hildreth and Diana Marver in the preparation of this manuscript. We also wish to thank Dr. Joseph Roland of the Vanderbilt Epithelial Biology Center Imaging Resource Core for providing Ariol SL-50 scanning analysis. This work was supported by pilot grants to DJA from the Vanderbilt-Meharry Center for AIDS Research (CFAR) (P30AI054999). 